\title{
Leukotoxin and its diol induce neutrophil chemotaxis through signal transduction different from that of fMLP
}

\author{
Y. Totani*, Y. Saito**, T. Ishizaki*, F. Sasaki**, S. Ameshima*, I. Miyamori*
}

\begin{abstract}
Leukotoxin and its diol induce neutrophil chemotaxis through signal transduction different from that of fMLP. Y. Totani, Y. Saito, T. Ishizaki, F. Sasaki, S. Ameshima, I. Miyamori. (C) ERS Journals Ltd 2000

ABSTRACT: When injected into animals, leukotoxin $(\mathbf{L x})$ causes acute lung injury which is associated with neutrophils infiltrating the lung tissues. However, the effect of Lx on neutrophils is still unknown, and recently it has been reported that $L x$ diol, a hydrolyzed metabolite, should be more potent than $\mathrm{Lx}$ in vitro. In this study, the authors examined the effect of $L x$ and its diol on human neutrophils by assessing their chemotactic response, expression of adhesion molecules, and production of peroxides.

Both $L x$ and its diol induced chemotaxis in human neutrophils via an involvement of pertussis toxin-sensitive G-proteins, but they did not influence the expression of adhesion molecules or the production of peroxides. Furthermore, Lx synergistically affected chemotaxis with $\mathrm{N}$-formyl-methionyl-leucyl-phenylalanine (fMLP), but not with endothelin-1.

Neutrophil chemotaxis induced by both $L x$ and its diol was inhibited by phosphatidylinositol-3-kinase (PI3-K) inhibitors, but not by protein tyrosine kinase (PTK) inhibitors or by protein kinase C (PKC) inhibitors, whereas fMLP-induced chemotaxis was inhibited by PTK inhibitors, but not by PI3-K inhibitors or by PKC inhibitors. These results suggest that neutrophil chemotaxis induced by both $\mathrm{Lx}$ and its diol involves pathways different from those induced by fMLP.

In conclusion, both leukotoxin and its diol metabolite induce chemotaxis in human neutrophils in a unique way and may act as important bioactive lipids when considering the pathological mechanism of acute lung injury. Eur Respir J 2000; 15: 75-79.
\end{abstract}

*Third Dept of Internal Medicine, Fukui Medical University, Fukui, Japan. **Division of Pulmonary and Allergy Medicine, Dept of Internal Medicine, Fujita Health University, Aichi, Japan.

Correspondence: Y. Totani, Third Department of Internal Medicine, Fukui Medical University, Mastuoka-cho, Fukui 910-11, Japan, Fax: 81776618111

Keywords: Acute lung injury

leukotoxin

leukotoxin diol

neutrophil chemotaxis

Received: November 241998

Accepted after revision September 71999

This work was supported by a Grant-inAid for Encouragement of Young Scientists (No. 0770420) and by Grants-in-Aid for Scientific Research (Nos. 07457145 and 06670605) from the Ministry of Education, Science and Culture of Japan.

Leukotoxin (Lx), a linoleate epoxide which is synthesized by neutrophils [1], appears in the bronchoalveolar lavage fluid (BALF) of patients with acute respiratory distress syndrome (ARDS) and causes acute lung injury when injected into rats [1]. However, the mechanisms of Lx-induced lung injury are still unclear. It has been reported previously [2-4] that Lx causes oedematous lung injury, which is inhibited by either nitric oxide synthase (NOS) inhibitors or superoxide dismutase in isolated perfused rat lungs, and that Lx augments superoxide anion $\left(\mathrm{O}_{2}{ }^{-}\right]$production by human pulmonary artery endothelial cells [15]. Polymorphonuclear neutrophils (PMNs) infiltrating the perivascular lesions and alveoli were observed after an intravenous injection of Lx in rats [6].

Neutrophils are now viewed as a critical factor in acute lung injury and studying the mechanisms by which they are involved is very important for a better understanding of this condition [7]. It was hypothesized that Lx may activate neutrophils, and that these activated neutrophils and neutrophil-derived oxygen radical species participate in Lx-induced lung injury. In the present study, the hypothesis was tested by assessing Lx chemotactic response, the expression of adhesion molecules, and the production of peroxides in neutrophils. Additionally, the signal transduction pathways involved in these effects of neutrophils treated with Lx were examined, and the study tested whether Lx acted synergistically with other chemoattractants.
Recently, MoghadDam et al. [8] reported that Lx should be regarded as a protoxin corresponding to the more toxic diol. Therefore the current study also examined the effect of Lx diol on the function of neutrophils using the same methods as for Lx.

\section{Materials and methods}

\section{Isolation of human polymorphonuclear neutrophils}

Human blood was collected in heparinized syringes from healthy adults. PMNs were isolated by Ficoll-Hypaque (Sigma, St Louis, MO, USA) centrifugation [9]. The cells were washed with Hank's balanced salt solution (HBSS), resuspended in HBSS, and adjusted to a concentration of $2 \times 10^{6}$ cells $\cdot \mathrm{mL}^{-1}$. Cell viability was tested by a Trypan blue dye exclusion test and $>90 \%$ were found to be alive.

\section{Chemotaxis chamber assay}

The effect of Lx (Cayman, Ann Arbor, MI, USA) or its diol (a gift from B.D. Hammock, University of California, Davis, CA, USA) on chemotaxis was measured by utilizing a chemotaxis chamber (Neuro Probe, Cabin John, MD, USA) [10]. The lower well was filled with $150 \mu \mathrm{L}$ of 
chemoattractant or HBSS and covered with a polyvinylpyrrolidone (PVP)-free polycarbonate filter (pore diameter, $3 \mu \mathrm{M}$; Neuro Probe). One hundred microlitres of PMN suspension was added to each upper well. For the chemotaxis assay, either Lx $\left(10^{-9}-10^{-5} \mathrm{M}\right)$, Lx diol $\left(10^{-10}-10^{-5}\right.$ $\mathrm{M})$, or $N$-formyl-methionyl-leucyl-phenylalanine (fMLP) (Peninsula, Belmont, CA, USA) as a positive control, or HBSS as a negative control, was added to each lower well. For the chemokinesis assay, the agents were added to both the upper well, which contained the PMN suspension, and the lower well. After incubation for 45 min at $37^{\circ} \mathrm{C}$, the filter was removed and the cells remaining on the upper surface of the filter were gently wiped off with a Filter wiper (Neuro Probe). The cells retained on the filter were fixed and stained with DiffQuik (International Reagents, Kobe, Japan). The number of cells having migrated was counted at five different filter sites using a $\times 100$ objective lens by means of lightmicroscopy.

\section{Assay of adhesion molecules and peroxides}

The surface expression grade of the adhesion molecules (CD11b and CD18) was determined by flow cytometry. The PMN suspensions were incubated either with Lx $\left(10^{-8}-10^{-5}\right.$ $\mathrm{M})$, Lx diol $\left(10^{-8}-10^{-5} \mathrm{M}\right)$, or fMLP $\left(10^{-7} \mathrm{M}\right)$ as a positive control, or with HBSS as a negative control, for $30 \mathrm{~min}$. To the cells were added either fluorescein isothiocyanate (FITC)-labelled CD11b or CD18 monoclonal antibody (Coulter, Tokyo, Japan) on ice, and then incubated for 45 min in the dark. The cells were washed with phos-phatebuffered saline (PBS) without $\mathrm{Ca}^{2+}$ or $\mathrm{Mg}^{2+}$, fixed with $0.5 \%$ paraformaldehyde, and then analysed by an EPICS XL flowcytometer (Coulter). The production of peroxides was also determined by flow cytometry [11]. After incubation with either Lx $\left(10^{-8}-10^{-5} \mathrm{M}\right)$, Lx diol $\left(10^{-8}-10^{-5} \mathrm{M}\right)$, or fMLP $\left(10^{-7} \mathrm{M}\right)$ as a positive control, or HBSS as a negative control, the PMN suspensions were added to $10 \mu \mathrm{L}$ of $5 \mathrm{mM}$ 2,7-dichlorofluorescin diacetate (DCFH-DA) (Eastman Kodak, Rochester, NY, USA) and then incubated for 30 min at $37^{\circ} \mathrm{C}$. The cells were washed with $\mathrm{Ca}^{2+}$ and $\mathrm{Mg}^{2+}$-free PBS, fixed with $0.5 \%$ paraformaldehyde, and then analysed by the flowcytometer.

Studies with inhibitors and evaluation of synergism with other chemoattractants

In the chemotaxis chamber assay, the following inhibitors were used to study the chemotaxis signal transduction pathway. Before treating the PMNs with or without Lx, its diol, or fMLP, they were incubated with inhibitors under the following conditions: pertussis toxin (Sigma) (0.1-20 $\left.\mu \mathrm{g} \cdot \mathrm{mL}^{-1}, 60 \mathrm{~min}, 37^{\circ} \mathrm{C}\right)$; Herbimycin A (Sigma) (1-10 $\mu \mathrm{g} \cdot \mathrm{mL}^{-1}, 60 \mathrm{~min}, 37^{\circ} \mathrm{C}$ ); Erbstatin (Funakoshi, Tokyo, Japan) (1-15 $\left.\mu \mathrm{g} \cdot \mathrm{mL}^{-1}, 60 \mathrm{~min}, 37^{\circ} \mathrm{C}\right) ; \mathrm{H}-7$ (Sigma) $(5-50$ $\left.\mu \mathrm{M}, 10 \mathrm{~min}, 37^{\circ} \mathrm{C}\right)$; Calphostin C (Sigma) (1-1000 nM, 10 min, $37^{\circ} \mathrm{C}$ ) [10]; Wortmannin (Sigma) (10-200 nM, 15 min, $37^{\circ} \mathrm{C}$ ); and LY294002 (Sigma) (1-10 $\mu \mathrm{M}, 10 \mathrm{~min}$, $\left.37^{\circ} \mathrm{C}\right)[12]$

In the chemotaxis chamber assay, $N^{\mathrm{G}}$-mono-methyl-Larginine (L-NMMA) (Calbiochem, La Jolla, CA, USA), a NOS inhibitor, was used to examine whether endogenous
NO affects the neutrophil chemotaxis induced by Lx. Before treating PMNs with $\mathrm{Lx}\left(10^{-6} \mathrm{M}\right)$, PMNs were incubated with L-NMMA $(200 \mu \mathrm{M}-1 \mathrm{mM})$ for $40 \mathrm{~min}$ at $37^{\circ} \mathrm{C}$.

To assess possible synergism with other chemoattractants, the lower well of the chemotaxis chamber was filled with a combination of Lx $\left(10^{-8} \mathrm{M}\right)$ and either fMLP $\left(10^{-8}\right.$ M) or endothelin-1 (ET-1) (Peptide Institute, Osaka, Japan) $\left(10^{-9} \mathrm{M}\right)$ at their respective threshold concentrations. These two chemoattractants were used since fMLP is a wellknown chemoattractant and ET-1 synergistically works with Lx to promote oedematous lung injury in isolated perfused rat lungs [13, 14].

\section{Statistics}

Data are presented as mean \pm SEM with $\mathrm{n}$ values representing the number of different experiments. Differences between groups were tested by a Student's paired t-test. Differences were considered significant when $p<0.05$.

\section{Results}

Lx at $10^{-7}-10^{-6} \mathrm{M}$ significantly induced chemotaxis in a dose-dependent manner (fig. 1a). The chemotactic effect of Lx was maximal at a concentration of $10^{-6} \mathrm{M}$. Lx diol at $10^{-9}-10^{-7} \mathrm{M}$ also induced significant chemotaxis (fig. 1b). The chemotactic effect of Lx diol was maximal at a concentration of $10^{-8} \mathrm{M}$ and showed a classic bell-shaped curve. Interestingly, Lx diol was $\sim 100$ times more potent than Lx in terms of the chemotaxis of PMNs. Both Lx and its diol slightly induced chemokinesis (fig. 1). The concentration of the substances used did not affect cell viability during the experiments.

fMLP induced both the expression of the adhesion molecules (CD11b and CD18) and production of the peroxides (table 1). However, neither Lx nor its diol induced the expression of adhesion molecules or the production of peroxides (table 1).

Chemotaxis induced by Lx, its diol, or fMLP was inhibited by pertussis toxin (fig. 2). The inhibition was more potent in the former two than in the latter one and more susceptible to a low dose of pertussis toxin in Lx than its diol, suggesting that both Lx and its diol induce chemotaxis via a pertussis toxin-sensitive G-protein [15]. Both Lx and its diol-induced chemotaxis were inhibited by the phosphatidylinositol-3-kinase (PI3-K) inhibitors, Wortmannin and LY294002 [12] (fig. 3a), but not by the protein kinase $\mathrm{C}$ (PKC) inhibitors, Calphostin $\mathrm{C}$ or $\mathrm{H}-7$ [10] (fig. 3b), nor by the protein tyrosine kinase (PTK) inhibitors, Herbimycin A or Erbstatin (fig. 3c). fMLPinduced chemotaxis was inhibited by the PTK inhibitors, but not by the PI3-K inhibitors or PKC inhibitors (fig. 3).

Neutrophil chemotaxis induced by Lx $\left(10^{-6} \mathrm{M}\right)$ was not inhibited by L-NMMA ( $200 \mu \mathrm{M}-1 \mathrm{mM})$ (data not shown).

The combination of Lx $\left(10^{-8} \mathrm{M}\right)$ and fMLP $\left(10^{-8} \mathrm{M}\right)$, at their respective threshold concentrations, significantly induced chemotaxis (fig. 4). However, the combination of Lx $\left(10^{-8} \mathrm{M}\right)$ and ET-1 $\left(10^{-9} \mathrm{M}\right)$ did not induce chemotaxis. 

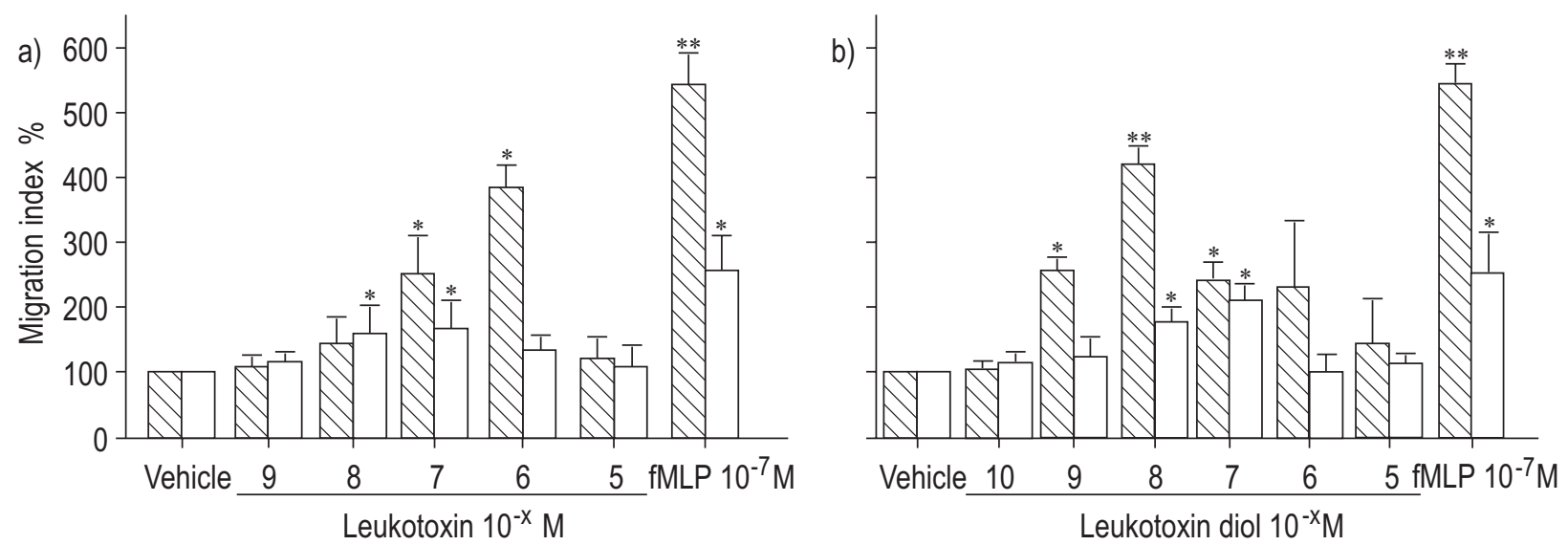

Fig. 1. - Effect of leukotoxin (Lx) (a), Lx diol (b), or $N$-formyl-methionyl-leucyl-phenylalanine (fMLP) (a and b) on chemotaxis and chemokinesis. Data are expressed as percentage of control and represent mean \pm SEM. $(n=5)$. $*$ : $p<0.05 ; *^{*}: \mathrm{p}<0.01$, versus vehicle. $\mathbb{\mathbb { N }}:$ chemotaxis; $\square:$ chemokinesis.

\section{Discussion}

Though neutrophils are now viewed as a critical factor in acute lung injury [7], there have been no reports examining the effect of Lx on neutrophil functions. In this study, it was found that Lx and its diol (with more potency than the latter) caused neutrophil chemotaxis, via a pathway involving a G-protein, and slight chemokinesis, but neither induced expression of adhesion molecules or production of peroxides. In keeping with a previous report that a large amount of Lx accompanied with many neutrophils was observed in BALF of patients with ARDS [1], and that neutrophils infiltrated into the perivascular lesions and alveoli after an intravenous injection of Lx in rats [6], it was considered that Lx-induced neutrophil chemotaxis plays a role in the mechanism of neutrophil recruitment to lung tissues in acute lung injury. Furthermore, the combination of Lx and fMLP, at their respective threshold concentrations, significantly induced chemotaxis. Thus, Lx may synergistically take a role with other chemoattractants in the recruitment of neutrophils.

Recently, it was reported that Lx diol, a hydrolyzed metabolite [16], was more toxic than Lx in vitro [8, 17]. The present study indicates that Lx diol induces chemotaxis at 100 -fold lower concentrations $\left(10^{-9}-10^{-7} \mathrm{M}\right)$ than Lx, thus confirming the higher potency of Lx diol $[8,17]$. However, because a 45 min exposure time is too short for Lx to be transformed into Lx diol [8], the current results also indicate that Lx, per se, is capable of inducing che-

Table 1. - Effect of leukotoxin (Lx) and Lx diol on the induction of adhesion molecules and peroxide production in human polymorphonuclear neutrophils

\begin{tabular}{lcccc}
\hline & Conc. & CD11b & CD18 & Peroxide \\
\hline Vehicle & & 100.0 & 100.0 & 100.0 \\
Lx & $10^{-6} \mathrm{M}$ & $93.7 \pm 5.2$ & $101.7 \pm 6.0$ & $74.3 \pm 15.1$ \\
& $10^{-8} \mathrm{M}$ & $101.1 \pm 3.6$ & $101.6 \pm 6.1$ & $94.1 \pm 9.5$ \\
Lx diol & $10^{-6} \mathrm{M}$ & $105.6 \pm 7.2$ & $103.2 \pm 4.1$ & $91.2 \pm 11.5$ \\
& $10^{-8} \mathrm{M}$ & $109.1 \pm 4.6$ & $103.2 \pm 3.6$ & $96.9 \pm 9.4$ \\
fMLP & $10^{-7} \mathrm{M}$ & $167.0 \pm 16.1^{*}$ & $150.9 \pm 7.6^{*}$ & $254.5 \pm 28.5^{*}$ \\
\hline
\end{tabular}

Data are expressed as percentage of control and represent mean \pm SEM. $(n=5) . *$ : $p<0.01$ versus vehicle. Conc.: concentration. motaxis in neutrophils at pathophysiological concentrations. Furthermore, because the Lx employed in the current study contained no trace amount of Lx diol, it is suggested that both Lx and its diol may act cooperatively in acute lung injury.

Signal transduction pathways different from those activated by fMLP have been reported in neutrophil migration $[10,12,15,18,19]$. Therefore, the authors analysed the signal transduction pathways involved in the chemotaxis of neutrophils treated with Lx or its diol by using the inhibitors of G-protein, PKC, PTK, and PI3-K.

Chemotaxis induced by Lx or its diol was inhibited by pertussis toxin, suggesting that pertussis toxin sensitive Gproteins, which have been reported to be important for neutrophil migration induced by fMLP, are also involved in the chemotaxis of neutrophils induced by both Lx and its diol $[10,15]$. The current results showing that fMLPinduced chemotaxis is inhibited by PTK inhibitors, but not by PKC inhibitors or PI3-K inhibitors, confirmed previous studies $[10,18,19]$. However, the finding that both Lx and its diol-induced neutrophil chemotaxis are inhibited by PI3-K inhibitors, but not by PTK or PKC inhibitors, suggests that chemotaxis of human neutrophils

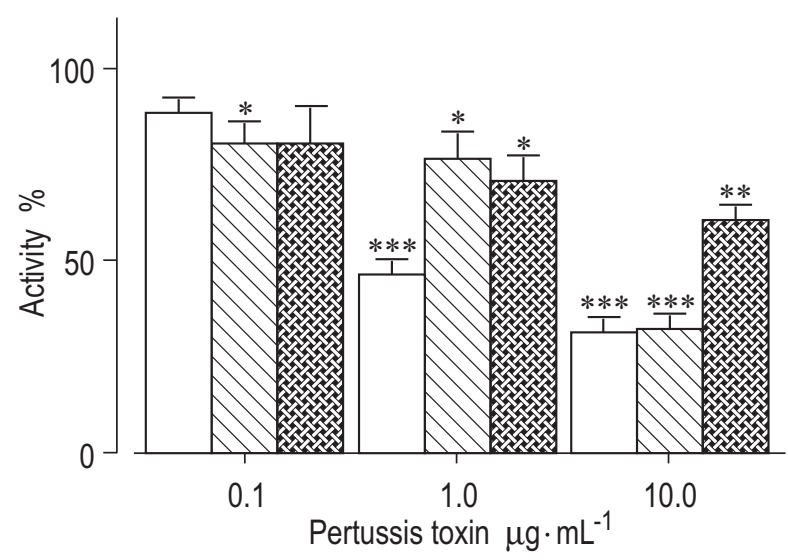

Fig. 2. - Effect of pertussis toxin on leukotoxin ( $\mathrm{Lx})$, Lx diol, or $\mathrm{N}$-formyl-methionyl-leucyl-phenylalanine (fMLP)-induced chemotaxis. Data are expressed as percentage of control without pertussis toxin and represent mean \pm SEM. $(\mathrm{n}=5)$. ${ }^{*}: \mathrm{p}<0.05 ;{ }^{* *}: \mathrm{p}<0.01 ; * * *: \mathrm{p}<0.001$, versus vehicle. $\square$ : Lx; $\mathbb{\mathbb { N }}$ : Lx diol; 丽 : fMLP. 

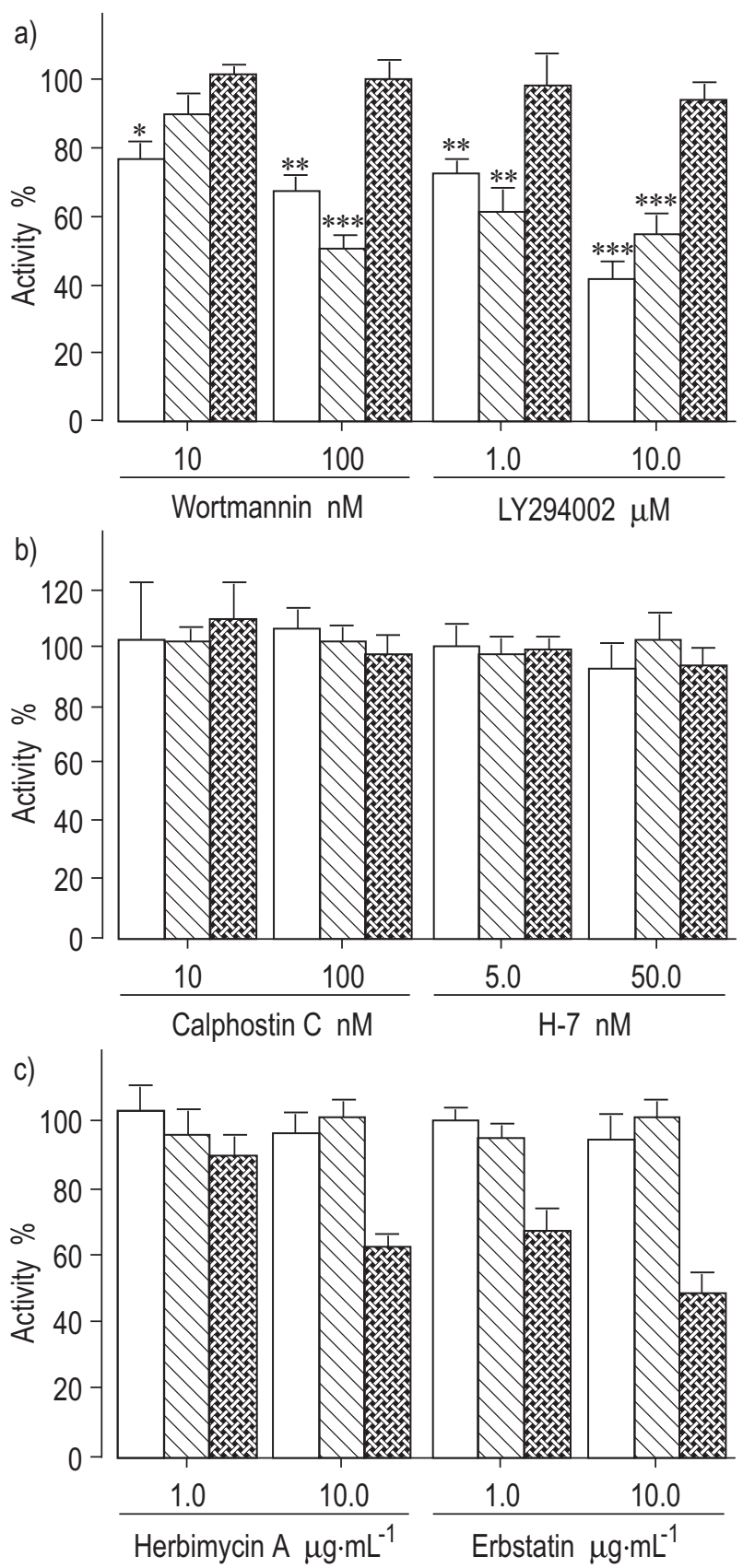

Fig. 3. - Effect of inhibitors on the chemotaxis induced by leukotoxin (Lx), Lx diol, or $N$-formyl-methionyl-leucyl-phenylalanine (fMLP). a) phosphatidylinositol-3-kinase (PI3K)-inhibitors, Wortmannin or LY294002 ; b) protein kinase $\mathrm{C}$ (PKC)-inhibitors, Calphostin $\mathrm{C}$ or $\mathrm{H}-7$; c) protein tyrosine kinase (PTK)-inhibitors, Herbimycin A or Erbstatin. Data are expressed as percentage of control without inhibitors and represent mean \pm SEM. $(\mathrm{n}=5) .{ }^{*}: \mathrm{p}<0.05 ; * *$ : $\mathrm{p}<0.01 ; * * *$ : $\mathrm{p}<0.001$, versus vehicle. $\square$ : Lx; $\mathbb{\mathbb { N }}$ : Lx diol; : fMLP.

induced by both Lx and its diol involve a pathway that differs from that activated by fMLP. This difference may be the reason that Lx and its diol did not induce an expression of adhesion molecules nor the induction of peroxides in the neutrophils, unlike fMLP.

In conclusion, both leukotoxin and its diol metabolite induce chemotaxis of human neutrophils in a unique way and may act as important bioactive lipids when considering the pathological mechanism of acute lung injury.

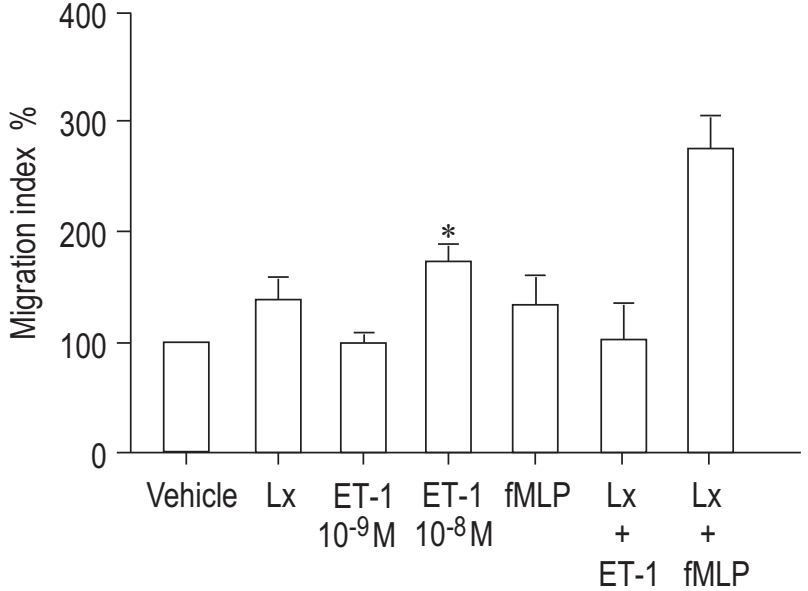

Fig. 4. - Effect of vehicle, leukotoxin (Lx) $\left(10^{-8} \mathrm{M}\right)$, endothelin-1 (ET1) $\left(10^{-9} \mathrm{M}\right)$, ET-1 $\left(10^{-8} \mathrm{M}\right)$, fMLP $\left(10^{-8} \mathrm{M}\right), \mathrm{Lx}\left(10^{-8} \mathrm{M}\right)+\mathrm{ET}-1\left(10^{-9}\right.$ $\mathrm{M})$, or $\operatorname{Lx}\left(10^{-8} \mathrm{M}\right)+\mathrm{fMLP}\left(10^{-8} \mathrm{M}\right)$ on chemotaxis. Data are expressed as percentage of control and represent mean $\pm \operatorname{SEM}$. $(n=5){ }^{*}: \mathrm{p}<0.01$ versus vehicle.

Acknowledgements. The authors are grateful to B.D. Hammock for supplying the leukotoxin diol.

\section{References}

1. Ozawa T, Sugiyama S, Hayakawa M, et al. Existence of leukotoxin 9,10-epoxy-12-octadecenoate in lung lavages from rats breathing pure oxygen and from patients with the adult respiratory distress syndrome. Am Rev Respir Dis 1988; 137: 535-540.

2. Ishizaki T, Shigemori K, Nakai T, et al. Leukotoxin, 9,10epoxy-12-octadecenoate causes edematous lung injury via activation of vascular nitric oxide synthase. $A m J$ Physiol 1995; 269: L65-L70.

3. Sakai T, Ishizaki T, Ohnishi T, et al. Leukotoxin, 9,10epoxy-12 octadecenoate inhibits mitochondrial respiration of isolated perfused rat lung. Am J Physiol 1995; 269: L326-L331.

4. Sakai T, Ishizaki T, Nakai T, et al. Role of nitric oxide and superoxide anion in leukotoxin-, 9,10 epoxy-12-octadecenoate,-induced mitochondrial dysfunction. Free Radic Biol Med 1996; 20: 607-612.

5. Ameshima S, Ishizaki T, Demura Y, et al. Production of superoxide anion and cell injury induced in human pulmonary artery endothelial cells by leukotoxin. Am J Respir Crit Care Med 1997; 155: A241.

6. Jia-ning H, Taki F, Sugiyama S, et al. Neutrophil-derived epoxide, 9,10-epoxy-12-octadecenoate, induces pulmonary edema. Lung 1988; 166: 327-337.

7. Canonico AE, Brigham KL. Biology of acute injury. In: Crystal RG, West JB, eds. The Lung. Philadelphia, PA, USA. Lippincott-Raven Publishers, 1997; pp. 24752491.

8. Moghaddam MF, Grant DF, Cheek JM, Greene JF, Williamson KC, Hammock BD. Bioactivation of leukotoxins to their toxic diols by epoxide hydrolase. Nat Med 1997; 3: $562-566$.

9. Kapp A, Zeck-Kapp G, Danner M, Lunger TA. Human granulocyte-macrophage colony stimulating factor: an effective direct activation of human polymorphonuclear neutrophilic granulocytes. J Invest Dermatol 1988; 91: $49-55$. 
10. Harakawa N, Sasada M, Maeda A, et al. Random migration of polymorphonuclear leukocytes induced by GMCSF involving a signal transduction pathway different from that of fMLP. J Leukoc Biol 1997; 61: 500-506.

11. Bass DA, Parce JW, Dechatelet LR, Szejda P, Seeds MC, Thomas M. Flow cytometric studies of oxidative product formation by neutrophils: a graded response to membrane stimulation. J Immnol 1983; 130: 1910-1917.

12. Coffer PJ, Geijsen N, M'rabet L, et al. Comparison of the roles of mitogen-activated protein kinase and phosphatidylinositol 3-kinase signal transduction in neutrophil effector function. Biochem J 1998; 329: 121-130.

13. Ishizaki T, Shigemori K, Nakai T, et al. Endothelin-1 potentiates leukotoxin-induced edematous lung injury. $J$ Appl Physiol 1995; 79: 1106-1111.

14. Ishizaki T, Shigemori K, Ameshima $\mathrm{S}$, et al. Protective effects of $\mathrm{BQ}-123$, an $\mathrm{ET}_{\mathrm{A}}$ receptor antagonist, against leukotoxin-induced injury in rat lungs. Am J Physiol 1996; 271: L459-L463.
15. Boonen GJ, de-Koster BM, VanSteveninck J, Elferink JG. Neutrophil chemotaxis induced by the diacylglycerol kinase inhibitor R59022. Biochim Biophys Acta 1993; 1178: 97-102.

16. Moghaddam MF, Motoba K, Borhan B, Pinot F, Hammock BD. Novel metabolic pathways for linoleic and arachidonic acid metabolism. Biochim Biophys Acta 1996; 1290: 327-339.

17. Moran JH, Weise R, Schnellmann RG, Freeman JP, Grant DF. Cytotoxicity of linoleic acid diols to renal proximal tubular cells. Toxicol Appl Pharmacol 1997; 146: 53-59.

18. Thelen M, Uguccioni M, Bosiger J. PI3-kinase-dependent and independent chemotaxis of human neutrophil leukocytes. Biochem Biophys Res Commun 1995; 217: 12551262.

19. Yasui K, Yamazaki M, Miyabayashi M, Tsuno T, Komiyama A. Signal transduction pathway in human polymorphonuclear leukocytes for chemotaxis induced by chemotactic factor. J Immunol 1994; 152: 5922-5929. 